\title{
Histopathological Evaluation of Urothelial Carcinomas in Transurethral Resection Urinary Bladder Tumor Specimens: Eight Years of Single Center Experience
}

\author{
Ali Koyuncuer
}

\begin{abstract}
Background: Urothelial carcinoma (UC) is a malignant neoplasm that most commonly occurs in the urinary bladder. The primary aim of this study was to evaluate the clinicopathologic features, recurrence and progression in patients with bladder urothelial cancer. Materials and Methods: The medical records of patients diagnosed with UC in the state pathology laboratory between January 2006 and July 2014 were retrospectively included. Carcinomas were categorized according to age, gender, histologic grade, tumor configuration, pathologic staging, recurrence status, and progression. Results: A total of 125 (113 men, 12 women) patients were examined. The mean age was 65.9 years and the male-to-female urothelial cancer incidence ratio was 9.4:1. Low-grade UCs were observed in $85(68 \%)$ and high-grade in $40(32 \%)$. A papillary tumor pattern was observed in $67.2 \%$ of the UCs. Cases were classified with the following pathological grades: $34(27.2 \%)$ cases of pTa, $70(56 \%)$ of pT1, and $21(16.8 \%)$ of pT2. Recurrence occurred in $27(21.6 \%)$ patients. Ten progressed to a higher stage (pT1 to pT2), and three cases to higher grade (low to high). We also analyzed the results separately for $70(56 \%)$ patients 65 years of age and older. Conclusions: With early detection and diagnosis of precursor lesions in older patients, by methods such as standard urologic evaluation, urinary cytology, ultrasound scanning and contrast urography, and cystoscopy, in addition to coordinated efforts between pathologists and urologists, early diagnosis may reduce the morbidity and mortality of patients with urothelial carcinoma.
\end{abstract}

Keywords: Age - bladder cancer - histologic grade - gender - pathologic stage - progression - recurrence

Asian Pac J Cancer Prev, 16 (7), 2871-2877

\section{Introduction}

Worldwide, urinary bladder cancer is the seventh most common malignancy of the urinary tract (Lopez-Beltran et al., 2004)and accounts for approximately $3.2 \%$ of all cancers (Parkin et al., 1999). An estimated 74,690 new cases and 15,580 deaths will occur in 2014 in the United States (Siegel et al., 2014). These tumors usually occur in the geriatric population with a male predominance (male to female, ratio; $3.5: 1$ ). The median age at the time of diagnosis is 69 years old for men and 71 years old for women (Parkin et al., 1999; Taylor et al., 2009). Epidemiologic studies have reported important risk factors for urinary bladder carcinomas are cigarette-smoking and occupational exposure to aromatic amines. Other risk factors include biomarker/genetic susceptibility, fluid consumption and water pollutants, environmental pollution (arsenic), gender (male), socioeconomic status, coffee consumption, urinary tract disease (stone, inflammation), schistosoma haematobium infection, exposure to ionizing radiation, cyclophosphamide, and other pharmaceutical agents (phenacetin) (Negri et al., 2007; Burger et al., 2013). The most common symptoms and signs are haematuria, pain, dysuria, less often appetite, weight loss, respiratory findings, renal insufficiency either from clinical manifestations locally, advanced or metastatic disease (DeSouza et al., 2014; Shephard et al., 2012). The two man categories of urothelial carcinoma include noninvasive papillary UC, and invasive UC. Urothelial carcinoma (UC) patients have at presentation a limited mucosa-submucosa invasion (superficial, also known nonmuscle invasive) in $80 \%$ of patients, as dictated by staging of the primary tumor, pTa and pT1 (Cheng et al., 2012) while 10-20\% of newly diagnosed patients present with invasive carcinoma (Eble et al., 2013). Urothelial carcinoma (so-called transitional cell) is the most common type of urinary bladder carcinoma (Lopez-Beltran et al., 2004). The major prognostic factor for urothelial tumors is the histologic grade. A histologic system for classifying urothelial tumors has been proposed; papilloma, papillary neoplasm of low malignant potential, low-grade papillary carcinoma, and high-grade papillary carcinoma (Rosai, 2011). The five year survival rate for patients with noninvasive UC treated with cystectomy is about $78.6 \%$, while UC with invasion of the detrusor muscle is approximately 6-40\% (Skinner, 
Table 1. Distribution of Histologic Grade, Pathologic Stage, Mean Age and Gender in Patients with Urothelial Carcinomas $(\mathbf{n}=\mathbf{1 2 5})$ of the Urinary Bladder

\begin{tabular}{|c|c|c|c|c|c|}
\hline \multicolumn{3}{|c|}{ Histologic grade } & \multicolumn{2}{|c|}{ Gender } & \multirow{2}{*}{$\begin{array}{c}\text { Mean Age (Years) } \\
\text { Male/Female }\end{array}$} \\
\hline & & & Male & Female & \\
\hline \multirow[t]{4}{*}{ Low-grade } & Pathologic Staging & $\mathrm{pTa}$ & $27(31.80 \%)$ & $5 \quad(5.90 \%)$ & $63.9 / 64.0$ \\
\hline & & pT1 & $47(55.30 \%)$ & $4(4.70 \%)$ & $66.3 / 74.0$ \\
\hline & & pT2 & $2(2.40 \%)$ & $0 \quad(0.00 \%)$ & $75.0 / 0$ \\
\hline & & Total & $76(89.40 \%)$ & $9(10.60 \%)$ & $68.4 / 69.0$ \\
\hline \multirow[t]{4}{*}{ High-grade } & Pathologic Staging & $\mathrm{pTa}$ & $1 \quad(2.50 \%)$ & $1 \quad(2.50 \%)$ & $80.0 / 75.0$ \\
\hline & & pT1 & $19(47.50 \%)$ & $0 \quad(0.00 \%)$ & $63.4 / 0$ \\
\hline & & pT2 & $17(42.50 \%)$ & $2(5.00 \%)$ & $66.9 / 72.0$ \\
\hline & & Total & $37(92.50 \%)$ & $3(7.50 \%)$ & $70.1 / 73.5$ \\
\hline
\end{tabular}

1977). The recurrence rate after transurethral resection is $30-60 \%$, and $10-15 \%$ of patients develop progression with advanced disease (invasion of the muscle) (Vedder et al., 2014). The aim of the present study was to clarify the histopathologic features and patient characteristics of UC diagnosed in urinary bladder specimens.

\section{Materials and Methods}

\section{Study design}

In this retrospective study, four micrometer thick formalin-fixed and paraffin-embedded sections created from transurethral resection of bladder tumor (TURBT) specimens of patients with urothelial carcinoma wereexamined. The specimens were stained with hematoxylineosin. We excluded non-invasive papillary urothelial neoplasm of low malignant potential, squamous cell carcinoma, adenocarcinoma, small cell carcinoma and other neoplasms. All samples were obtained from State Hospital's pathology laboratory between January 2006 and July 2014. All cases were categorized according to age, gender, histologic grade, tumor configuration, microscopic tumor extension or pathologic staging (primary tumor, pT), recurrent tumors and patient age; $\leq 40$ years, 41 to 50 years, 51 to 60 years, 61 to 70 years, 71 to 80 years, and $>81$ years. Histologic grade was categorized as lowgrade or high-grade; pathologic staging was categorized as pTa, noninvasive papillary carcinoma; pT1, tumor invades subepithelial connective tissue (lamina propria); pT2, tumor invades muscularis propria (detrusor muscle) according to the classification of the College of American Pathologists (CAP), World Health Organization (WHO) 2004/ International Society of Urologic Pathology (ISUP) Consensus Classification and the tumor node metastasis (TNM) Classification of Malignant Tumours (UICC 2009) (Lopez-Beltran et al., 2004; Sobin et al., 2009; College of American Pathologists, (CAP) 2014).

\section{Statistical analyses}

Data were analyzed using Statistical Package for Social Sciences (SPSS) software (version 21.0 for Windows, IBM Corp. Released 2012. IBM SPSS Statistics for Windows, Armonk, NY: IBM Corp.). A student t-test was used to compare averages, a chi-square test was used to determine the dispersion of the two groups (low vs high-grade), and a Kruskal-Wallis-H test was used to determine the dispersion of pathologic staging group. All differences associated with a chance probability of 0.05 or
Table 2. Correlations between Pathologic Stage and Histologic Grade

\begin{tabular}{|c|c|c|c|c|}
\hline \multirow[b]{2}{*}{ Histologic Grade } & \multicolumn{3}{|c|}{ PathologicStage } & \multirow[t]{2}{*}{ Total } \\
\hline & pTa & pT1 & pT2 & \\
\hline Low-g & $32(25.6 \%)$ & $51(40$ & $(1.6 \%)$ & $85(68 \%)$ \\
\hline High-Grade & $2(1.6 \%)$ & $19(15.2 \%) 19$ & $(15.2 \%)$ & $40(32 \%)$ \\
\hline Total & $34(27.2 \%)$ & $70(56.0 \%) 21$ & [ $(16.8 \%)$ & $125(100 \%)$ \\
\hline
\end{tabular}

less were considered statistically significant. Continuous variables are presented as mean \pm standard deviation $(\mathrm{SD})$.

\section{Results}

\section{Patient characteristics}

A total of 125 carcinomas of the urinary bladder with histologic urothelial carcinoma (UC, transitional cell) in cases examined between January 2006 and July 2014 were reported.

\section{Age}

The mean age of the patients was $65.96 \pm 12.1$ years (range 37 to 88 years). The median age detected in men for UC was 65.58 years, and in women was 69.58 years. Distribution of cases among age groups was: $\leq 40$ years, $1.6 \%$; 41 to 50 years, $9.6 \%$; 51 to 60 years, $23.2 \%$; 61 to 70 years, $25.6 \%$; 71 to 80 years, $25.6 \%$; and $>81$ years, $14.4 \%$. Cases $\leq 60$ years of age comprised $34.4 \%$ of patients, while $\geq 61$ years of age comprised $65.6 \%$ of patients. No statistically significant difference was found between age or age groups and histologic grade or pathologic staging $(\mathrm{P}=0.968,0.318,0.986,0.394$, respectively).

\section{Gender}

There were 113 male (90.4\%) and 12 female (9.6\%) cases in this study. No differences could be shown between the histologic grade and pathologic staging with respect to gender $(\mathrm{P}=0.588,0.205$, respectively).

\section{Histologic grade}

Low-grade UCs (LGUCs) were detected in $85(68 \%)$ cases while high-grade UCs were identified in 40 (32\%) of the cases (see Table 1,2,3). LGUCs were encountered in 85 of 125 patients, including $32(37.6 \%)$ with pTa; HGUCs were encountered in all patients, including 19 (47.5\%), and 19 (47.5\%) with pT1 or pT2, respectively. A statistically significant correlation between histologic grade and pathological staging was observed $(\mathrm{P}=0.000)$. 
Table 3. Distribution of Histologic Grade, Pathologic Stage, and Age group in Patients with Urothelial Carcinomas $(\mathrm{n}=125)$ of the Urinary Bladder

\begin{tabular}{lllccc}
\hline Histologic Grade & & \multicolumn{2}{c}{ Age Group } & Total \\
& & & $<64$ year n $(\%)$ & $\geq 65$ year n $(\%)$ & $1 \%)$ \\
\hline Low-grade & Pathologic Stage & pTa & $15(17.6 \%)$ & $17(20 \%)$ & $32(37.6 \%)$ \\
& & pT1 & $23(27.1 \%)$ & $28(32.9 \%)$ & $51(60 \%)$ \\
& & pT2 & $0(0 \%)$ & $2(2.4 \%)$ & $2(2.4 \%)$ \\
High-grade & Total & pTa & $38(44,7 \%)$ & $47(55.3 \%)$ & $85(100 \%)$ \\
& Pathologic Stage & pT1 & $8(0 \%)$ & $2(5 \%)$ & $2(5 \%)$ \\
& & pT2 & $9(22.5 \%)$ & $11(27.5 \%)$ & $19(47.5 \%)$ \\
& & & $17(42.5 \%)$ & $23(57.5 \%)$ & $40(100 \%)$ \\
Total & Total & pTa & $15(12 \%)$ & $19(15.2 \%)$ & $34(27.2 \%)$ \\
& Pathologic Stage & pT1 & $31(24.8 \%)$ & $39(31.2 \%)$ & $70(56 \%)$ \\
& & pT2 & $9(7.2 \%)$ & $12(9.6 \%)$ & $21(16.8 \%)$ \\
& & & $55(44 \%)$ & $70(56 \%)$ & $125(100 \%)$ \\
\hline
\end{tabular}

\section{Tumor configuration}

The vast majority of tumors exhibited a papillary configuration $(67.2 \%)$, and less often a solid/nodule, or mixed (papillary and solid) configuration. Tumors with a predominantly papillary configuration were found in $88.2 \%$ of all patients in pTa, in $67.1 \%$ of all patients pT1, in $33.3 \%$ of all patients with pT2, in $78.8 \%$ of all patients with low-grade malignancy, and in $42.5 \%$ of all patients high-grade malignancy (Figures 1a, 1b). There was also a significant correlation between tumor configuration and histologic grade or pathologic staging $(\mathrm{P}=0.004,0.000$, respectively).

\section{Pathologic staging (primary Tumor, $p T$ )}

pTa (noninvasive papillary carcinoma) was observed in $34(27.2 \%)$ cases, pT1 (tumor invades subepithelial connective tissue (lamina propria)) and pT2 (tumor invades muscularis propria (detrusor muscle)) in 70 (56\%) cases and, $21(16.8 \%)$ patientscases, respectively. Thirty-four patients (27.2\%) found to havewith UC wereas classified diagnosed with noninvasive carcinoma, 91 (72.8\%) patients were diagnosed with (72.8\%) invasive carcinoma (lamina propria invasion, muscularis propriadetrusor muscle invasion) and $104(83.2 \%)$ patients (83.2\%) with nonmuscle invasive bladder cancer (Figure 1c). Of those, noninvasive carcinoma occurred in 34 of 125 patients (27.7\%), including 32 (25.6\%) LGUC, and two 2 (1.6\%) with HGUC. Invasive carcinoma was encountered in 91 of 125 patients (72.8\%), including $53(42.4 \%)$ with LGUC, and 38 (30.4\%) with HGUC. A correlation was detected between invasive status and histologic grade of tumor $(\mathrm{P}=0.000)$. Muscle invasive urothelial carcinoma was more common in males than in females $(15.2 \%$ and $1.6 \%, \mathrm{P}=0.052)$.

\section{Patient characteristics of recurrent tumors}

Recurrence was observed in 27 of the 125 (21.6\%) patients in all tumors; however, 98 (78.4\%) patients did not have a recurrence (Table 4 ). The mean age of patients was $67.4 \pm 12.6$ (range 37 to 86 ) years old. Recurrent UC was observed in $21(77.8 \%)$ of the males and in six $(22.2 \%)$ of the female cases in this study. There was a correlation or significant statistical association between recurrent tumors and gender $(\mathrm{P}=0.012)$. Low-grade UC had a recurrence

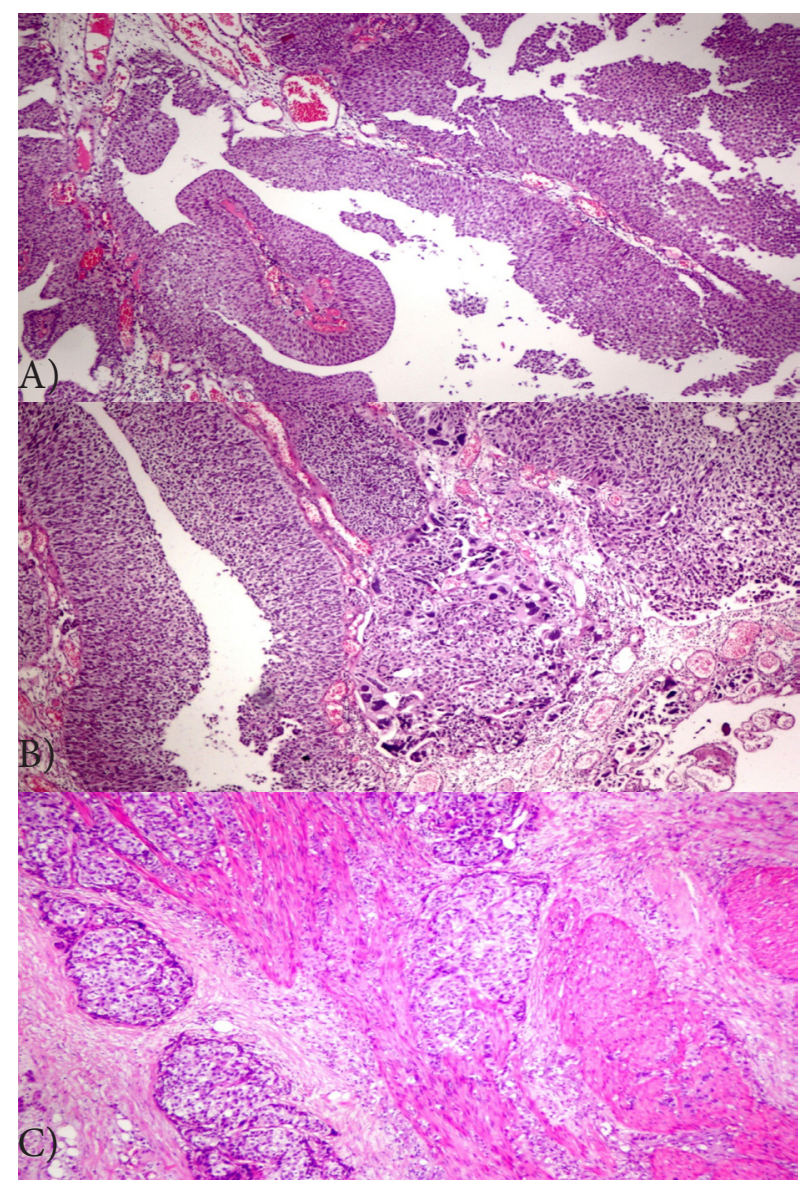

Figure 1. Urothelial Carcinomas. A) Low-grade papillary, Pathologic Staging, pTa;noninvasive (Hematoxylin-Eosin, original magnification $\times 40$ objective). B) High-grade urothelial carcinoma, papillary, Pathologic Staging, pT1; tumor invades subepithelial connective tissue-lamina propria (HematoxylinEosin, original magnification $\times 40$ objective). C) Pathologic Staging, pT2: Tumor invades muscularis propria (detrusor muscle) (Hematoxylin-Eosin, original magnification $\times 100$ objective)

in 19 cases $(70.4 \%)$ and high-grade UC had a recurrence in $8(29.6 \%)$. Tumor recurrences were observed in $\mathrm{pTa}$, pT1 and pT2 in 10 (37\%), 16 (59.3\%), 1 (3.7\%) case, respectively. There was a significant correlation between recurrent bladder cancer and pathologic staging $(\mathrm{P}=0.040)$. No statistically different relationship was found between 
recurrent disease and histologic grade of tumor $(\mathrm{P}=0.768)$. Twenty-three $(85.2 \%)$ patients had one recurrence, three $(11.1 \%)$ patients had two recurrences, and one $(3.7 \%)$ patient had three recurrences. The mean count of episodes was 1.18 .

\section{Recurrence of year}

Cases of UC were recorded during an eight-year period. Recurrence of UC within $\leq 1$ year following the first transurethral resection was seen in $15(55.6 \%)$ patients and $>1$ year in $12(44.4 \%)$ patients. The mean process time to first recurrence at follow-up was 11.9 months (minimum 1, maximum 36 months). Cases with UC recurrence had a rate of $81.5 \%$ at the 24 month followup period. All patients had no evidence of extravesical extension, no metastasis and no evidence of death. The association recurrence for the years and also pathologic staging were statistically significant $(\mathrm{P}=0.028)$. There was no correlation between recurrence for years and histologic grade $(\mathrm{P}=0.510)$.

\section{Stage or grade progression}

Ten patients (37\%, all total recurrent cases) were diagnosed as having tumour progression from pT1 carcinomas to pT2 carcinomas in later transurethral resections. Three patients showed progression from a low to high grade tumor. In two patients with tumour recurrence, the tumor progressed to muscle invasion.

\section{Older patients $(\geq 65$ years $)$}

We analyzed the results in 70 patients (56\%) 65 years of age and older. Low-grade and high-grade UC was found approximately in $67.1 \%$ and $32.9 \%$ of older patients, respectively. Nineteen patients $(27.1 \%)$ were pTa and 39 patients $(55.7 \%)$ were pT1 and 12 patients (17.1\%) were pT2. There were $69.6 \%$ (18/27 cases) with recurrent bladder carcinoma who were 65 years of age and older. No statistically significant relationship was found between older patients and histologic grade

Table 4. Clinicopathologic Features and Tumor Recurrences

\begin{tabular}{llcr}
\hline & & \multicolumn{2}{c}{ Age Group } \\
& & <64 yrs & $\geq 65$ yrs \\
& & $\mathrm{n}$ & $\mathrm{n}$ \\
\hline Gender & Male & 8 & 13 \\
& Female & 1 & 5 \\
Pathologic Staging & pTa & 4 & 6 \\
& pT1 & 5 & 11 \\
Histologic Grade & pT2 & 0 & 1 \\
Interval to recurrencemonths & Low-grade & 5 & 14 \\
& High-grade & 4 & 4 \\
& $<12$ months & 6 & 9 \\
Progression pT & $12-24$ months & 2 & 5 \\
& $>24$ months & 1 & 4 \\
Progression HG & No & 4 & 13 \\
& Progression & 5 & 5 \\
Muscularispropria & No & 9 & 15 \\
(detrusor muscle) & Progression & 0 & 3 \\
\hline
\end{tabular}

of tumor, pathologic staging and recurrence $(\mathrm{P}=0.819$, $0.939,0.210$, respectively). Analysis according to tumor histology showed a trend; the elderly ( $>81$ years) patients had outcomes were noted with low-grade histology 13 patients $(10.4 \%)$ and high-grade histology 5 patients (4\%). Four patients $(3.2 \%)$ were pTa and 9 patients $(7.2 \%)$ were pT1 and 5 patients (4\%) were pT2.

Adequacy of material to identify muscularis propria (detrusor muscle)

Muscularis propria (detrusor muscle) was identified in $97(77.6 \%)$ transurethral resection specimens in total and the muscularis propria was not identified in $28(22.4 \%)$ cases. The muscularis propria was absent in 1/28 (3.5\%) high-grade Ta and 18/28 (64\%) T1 specimens.

\section{Discussion}

Urothelial carcinoma is the most common carcinoma of the urinary bladder, accounting for more than $90 \%$ of all primary carcinomas (Lopez-Beltran et al., 2004). Urinary bladder cancer has the fourth highest new cases of cancer diagnosed in men in 2014 in the United States (Siegel et al., 2014) and the seventeenth most frequently diagnosed cancer in women worldwide (Kakehi et al., 2010). In European Union countries, the mortality rate in men is 7.2 to $6.1 / 100,000$ and in women approximately 1.3/100,000 (La Vecchia et al., 2010). The major risk factor for developing bladder cancer is cigarette smoking in a dose-dependent for both sexes (Burch et al., 1989), with an four-fold increased incidence compared to nonsmokers. Occupational polycyclic aromatic hydrocarbons are also responsible for approximately twenty percent of bladder cancer (Chu et al., 2013). Occupational hazards are one of several risks in the development of bladder cancer. An increased risk of urinary bladder carcinoma as an occupational risk for workers (eg, dye, rubber, leather, aluminum) have been reported (Silverman et al., 1992). The incidence of bladder cancer is predominantly seen more in men than women (Lippka et al., 2013). Investigations on this subject are numerous and clear data is available. Horstmann et al reported the incidence of urinary bladder cancer male-to-female ratio to be $2: 1$ (Horstmann et al., 2008), Hoke et al observed this ratio to be 1.33 to 1 (Hoke et al., 1999)while Quirk et al and Koyuncuer demonstrated the ratio of male-to-female patients to be 3.2:1, 3.76:1 respectively (Quirk et al., 2004). Thus, the ratio of male to women often differs between studies. Gupta et al reported a male to female ratio of 8.6:1 (Gupta et al., 2009). In our study, gender differences were seen in men more than women, with their onset being closer to those mentioned in the Gupta et al. In our study, we found, an overwhelming incidence in men, with a male:female ratio of 9.4:1. As far as we know, bladder cancer occurs almost exclusively in the geriatric population. Urinary bladder cancer tends to occur in the elderly (Hoke et al., 1999), but it does occur in young adults (Nomikos et al., 2011), and pediatric cases have also been reported (Stanton et al., 2013; Wang ZH et al., 2012). In a previous study, Horstmann et al. reported the mean age in males to be 62 , and females to be 67 ; Hoke 
et al observed the mean age in males to be 74.2 , and in women to be 67.3, Gupta et al,Quirk et al and Koyuncuer described that men age $60.2,65.3,62.9$ respectively (Hoke et al., 1999; Quirk et al., 2004; Horstmann et al., 2008; Gupta et al., 2009; Koyuncuer, 2013). In our study, urothelial carcinoma presentation had a mean age 65.9 (men, women mean age: 65.9 years vs 69 years, respectively) similar to that reported in the literature. In a recent study, researchers found $44.7 \%$ of UC cases to be a low-grade malignancy and $55.3 \%$ to be a high-grade malignancy (Gupta et al., 2009)and, Cheng and colleagues examined 105 transurethral resections of bladder tumor (TURBT) specimen and found $12.3 \%$ patients to have low-grade carcinoma $87.7 \%$ to have high-grade carcinoma (Cheng et al., 2000).

In our study, low-grade UC was seen in more than half of the cases, and thus our rates were different from the literature findings. The results were contrary to the neoplastic conditions of the urinary bladder of several previous published studies. Pan et al found low to high grade urothelial carcinoma that accounted for $46.6 \%$ and $39.4 \%$ in transurethral resection specimens, respectively (Pan et al., 2010). In another series, $77.8 \%$ of low grade papillary UC and $22.2 \%$ of high grade papillary urothelial carcinoma was observed (Koyuncuer, 2013). Oosterhuis et al reported a $75.8 \%$ of low grade papillary urothelial carcinoma, $24.2 \%$ high grade papillary urothelial carcinoma findings among 186 patients with urothelial carcinoma (Oosterhuis et al., 2002). In our study the rate observed is in accordance with the literature. Low-grade UC was seen in $85(68 \%)$ cases while $40(32 \%)$ cases were high-grade UCs. Low grade tumours, on the other hand, are associated with increased survival or longer cancer free intervals (Schapers et al., 1994). However, Jimenez et al demonstrated that the pathologic $\mathrm{T}$ stage was associated with the progression of invasive muscle carcinoma. The authors commented that the histologic grade is not a prognostic indicator for urothelial carcinoma to invade the muscle (Jimenez et al., 2000). The published results concerning a papillary tumor configuration in a given histologic grade have also been reported. Schned et al reported low-grade papillary lesions with a papillary configuration in $60 \%$ of tumors and high-grade papillary carcinomas account for $22.6 \%$ while non-papillary urothelial carcinoma accounts for $10.1 \%$. The authors also reported that a papillary configuration was seen in LGPUC which accounted for $93.7 \%$ in pTa, $6.3 \%$ in pT1 or pT2, in HGPUC which accounted for $35.1 \%$ in pTa, $64.9 \%$ in pT1 or pT2 (Schned et al., 2008). In our study, the papillary tumor configuration was seen at a rate similar to the literature. In our study, we found that the papillary growth pattern of the tumour was detected in $32.9 \%$ of pTa, in $45.9 \%$ of pT1 of all LGPUC and in $5 \%$ of pTa, in $20 \%$ of pT1, in $17.5 \%$ of pT 2 of all HGPUC. Mostofi et al argued that the growth pattern of the tumour serves as a prognostic factor. Papillary carcinoma throughout have a particularly favorable prognosis as compared to an infiltrating and mixed growth pattern (Mostofi et al., 1973). Tumor configuration with an infiltrative pattern also has a poor prognosis (Lopez-Beltran et al., 2004). Andius and colleagues showed that a stage $\mathrm{T} 1$ bladder cancer with a solid tumor configuration relationship has a higher risk of progression and poor prognosis (Andius et al., 2007). As previously noted, microscopic tumor extension (or pathologic staging) in TURBT specimens with bladder cancer has been reported, in $14.3 \%$ (15 cases) with pTa carcinoma, in $52.4 \%$ (55 cases) with pT1 carcinoma and $33.3 \%$ (35 cases) with pT2 carcinoma (Cheng et al., 2000). In our study, pT1 was seen in more than half of the cases, and thus our rates were similar to the literature findings. In our study, over seventy-seven percent of patients with invasive carcinoma are pT1 in contrast to twenty three percent of the patients with pT2. However, Choi et al observed pathologic staging in TURBT and cystectomy pathology results, in $41.5 \%$ with pTa carcinoma, in $21.5 \%$ with pT1 carcinoma and $14 \%$ with pT2 carcinoma (Choi et al., 2007). The recurrence and progression rate with respect to urinary bladder carcinoma is also variable in the published series. In a recent study by Chamie and colleagues, they observed bladder cancer recurrence rates of $39.1 \%$ and a rate of progression of $33 \%$. In the same study, the ten-year recurrence, progression and mortality was $74.3 \%, 33.3 \%$, and $12.3 \%$, respectively (Chamie et al., 2013). Superficial bladder cancer (pTa,pT1) has a documented recurrence rate of 50.8\% (Akagashi et al., 2006). Herr et al reported that low grade papillary bladder tumors show a mean number of recurrence episodes of 6.6 and observed a $67 \%$ recurrence rate. The authors observed a $17 \%$ progression rate grade or stage obtained in 215 cases treated (Herr et al., 2007).

In a literature review of several reports of low-grade papillary urothelial carcinoma, recurrence rates range from $34-72 \%$ and progression rates range from 4-10.5\%; for high-grade urothelial carcinoma, recurrence rates range from $43-74 \%$. Miyamoto et al recently reported that low-grade urothelial carcinoma had a higher rate $(53.8 \%)$ of recurrence. In the same study, the mean period at diagnosis of recurrence and progression was 13.9 months (2 months to 72 months) and 25.1 months, respectively (Miyamoto $\mathrm{H}$ et al., 2010). In another study, the recurrence and progression rate was $37 \%, 0 \%$ and $54 \%, 15 \%$ for low grade and high grade superficial bladder cancer, respectively (Millán-Rodríguez et al., 2000). Alsheikh and colleagues detected $48.2 \%$ of the low-grade papillary carcinoma in series recurred (four months to seven years) and two of the patients had a grade progression and two of the patients had invasion of the muscle (Alsheikh et al., 2001). On the other hand, in the literature it was shown that nonmuscle invasive bladder cancer is associated with a higher risk of one year and five year recurrence rate of $15-61 \%$ and $31-78 \%$, respectively (van der Heijden et al., 2009). In our series, $21.6 \%$ of the urothelial carcinomas exhibited a recurrence; recurrence tumors had a progression rate of $37 \%$. In our study, the recurrence, progression and mean course of time to first recurrence was seen at a rate in accordance with the literature. Several factors are thought to contribute to the development of this recurrence, including incomplete TUR, implantation of tumour cells, de novo or new tumor and other factors (van der Heijden et al., 2009; Bryan et al., 2010). We believe this is a reason for the early recurrence of urothelial carcinoma both for predominantly 


\section{Ali Koyuncuer}

incomplete TUR (residual tumour) and de novo or new tumours. More than half of the patients in our series had a recurrence occur at less than one year after transection. In a study of 30\% cases, patients' age ranged from 70-80 years, and $6 \%$ of patients were more than 80 years old having undergone a radical cystectomy for bladder cancer (Nielsen et al., 2007). In another study of 404 patients with radical cystectomy for bladder carcinoma, the median age was 74 years, with $87 \%$ of patients being between $70-79$ years and $13 \%$ being more than 80 years old (Figueroa AJ et al., 1998). Therefore, we found patients to be 65 years and older. On the other hand, patients aged 70 and older have an association with an increased risk of mortality in bladder cancer and in another study, cases aged 70 years and older had a death rate of $2.8 \%$ (Chamie et al., 2013; Figueroa et al., 1998). In our study, $22.4 \%$ of the cases did not demonstrate a muscularis propria in their TUR specimen and their rate was different compared to published studies. If muscle was present in the sample, 2 pT1 specimens were upstaged in all cases of progression. Maruniak et al reported the detrusor muscle was not identified in 51\% of cases (Maruniak et al., 2002).

In conclusion, the mortality rate is low today, owing to the fact that scientists are conducting extensive research and exhaustive investigations in this field. In our study, the overwhelming majority of urothelial carcinoma is composed of males with a peak incidence in the seventh decade. We believe that this difference likely is due to cigarette smoking and occupational, environmental risks or lifestyle factors. We observed that low-grade UCs and pathologic staging pT1 in all patients was detected in more than half of cases. These results in stage and grade of cancers probably are due to geographic, ethnic differences, tumour heterogeneity or other factors. Low grade carcinoma and stage pT1 bladder cancer were predominant in women. In this study, females were older at the age of detection and but had a low rate of muscle invasion and a lower rate of high grade carcinomas than males. Muscle invasive urothelial carcinoma was more common in males than in females.

This study included two patients in the third decade of life and three patients with low grade carcinomas that progressed to high carcinomas and two patients that progressed to muscle invasion in bladder cancer. However, the study demonstrated that two patients under the age of 40 years presented with pathologic stage Ta, noninvasive and low grade tumors. Recurrent tumors were found more commonly in male than female patients. The grade of recurrent disease was most frequently low grade. The patient's pathology stage or grade such as prognostic factors depends on incomplete or complete TUR. This made five-year survival, especially in the first year, easy to plan for recurrence or progression.

With early detection and diagnosis of precursor lesions in older patients, by methods such as standard urologic evaluation, urinary cytology, ultrasound scanning and contrast urography, and cystoscopy, in addition to coordinated efforts between pathologists and urologists, early diagnosis may reduce the morbidity and mortality of patients with urothelial carcinoma.

\section{References}

Akagashi K, Tanda H, Kato S,et al (2006). Recurrence pattern for superficial bladder cancer. Int J Urol, 13, 686-91.

Alsheikh A, Mohamedali Z, Jones E, et al (2001). Comparison of the WHO/ISUP classification and cytokeratin 20 expression in predicting the behavior of low-grade papillary urothelial tumors. World/Health Organization/Internattional Society of Urologic Pathology. Mod Pathol, 14, 267-72.

Amin MB, Delahunt B, Bochner BH, et al (2014). Protocol for the examination of specimens from patients with carcinoma of the Urinary Bladder. College of American Pathologists (CAP); 1996-2010. http://www.cap.org webcite.

Andius P, Johansson SL, Holmäng S (2007). Prognostic factors in stage $\mathrm{T} 1$ bladder cancer: tumor pattern (solid or papillary) and vascular invasion more important than depth of invasion. Urology, 70, 758-62.

Bryan RT, Collins SI, Daykin MC, et al (2010). Mechanisms of recurrence of Ta/T1 bladder cancer. Ann R Coll Surg Engl, 92, 519-24.

Burch JD, Rohan TE, Howe GR, et al (1989). Risk of bladder cancer by source and type of tobacco exposure: a casecontrol study. Int J Cancer, 44, 622-8.

Burger M, Catto JW, Dalbagni G, et al (2013). Epidemiology and risk factors of urothelial bladder cancer. Eur Urol, 63, 234-41.

Chamie K, Litwin MS, Bassett JC, et al (2013). Urologic Diseases in America Project. Recurrence of high-risk bladder cancer: a population-based analysis. Cancer, 119, 3219-27.

Cheng L, Neumann RM, Weaver AL, et al (2000). Grading and staging of bladder carcinoma in transurethral resection specimens. Correlation with 105 matched cystectomy specimens. Am J Clin Pathol, 113, 275-9.

Cheng L, Lopez-Beltran A, Bostwick DG (2012). Bladder cancer: general features,first ed.:A John Wiley \& Sons, Inc.,New Jersey, pp138-152.

Choi YL, Lee SH, Kwon GY,et al (2007). Overexpression of $\mathrm{CD} 24$ : association with invasiveness in urothelial carcinoma of the bladder. Arch Pathol Lab Med, 131, 275-81.

Chu H, Wang M and Zhang Z (2013): Bladder cancer epidemiology and genetic susceptibility. $J$ Biomed Res, 27, 170-8.

DeSouza K, Chowdhury S and Hughes S (2014): Prompt diagnosis key in bladder cancer. Practitioner, 258, 23-7.

Eble JN, Grignon DJ and Young RH (2013). Tumors of the urinary bladder and urethra. tumors of the urinary tract. In: Fletcher CDM editor,fourt ed.Elsevier Inc., Philadelphia (PA), pp614.

Figueroa AJ, Stein JP, Dickinson M, et al (1998). Radical cystectomy for elderly patients with bladder carcinoma: an updated experience with 404 patients. Cancer, 83, 141-7.

Gupta P, Jain M, Kapoor R, Muruganandham K, Srivastava A and Mandhani A (2009). Impact of age and gender on the clinicopathological characteristics of bladder cancer. Indian J Urol, 25, 207-10.

Herr HW, Donat SM and Reuter VE (2007): Management of low grade papillary bladder tumors. $J$ Urol, 178, 1201-5.

Hoke GP, Stone BA, Klein L and Williams KN (1999): The influence of gender on incidence and outcome of patients with bladder cancer in Harlem. J Natl Med Assoc, 91, 144-8.

Horstmann M, Witthuhn R, Falk M and Stenzl A (2008): Gender-specific differences in bladder cancer: a retrospective analysis. Gend Med, 5, 385-94.

Jimenez RE, Gheiler E, Oskanian P, et al (2000). Grading the invasive component of urothelial carcinoma of the bladder and its relationship with progression-free survival. Am J Surg Pathol, 24, 980-7. 
Kakehi Y, Hirao Y, Kim WJ, et al (2010). Bladder Cancer Working Group report. Jpn J Clin Oncol, 40, 57-64.

Koyuncuer A: Immunohistochemical expression of p63, p53 in urinary bladder carcinoma (2013). Indian J Pathol Microbiol, 56, 10-5.

Lopez-Beltran A, Bassi P, Pavone-Macaluso M and Montironi R (2004). Handling and pathology reporting of specimens with carcinoma of the urinary bladder, ureter, and renal pelvis. Eur Urol, 45, 257-66.

La Vecchia C, Bosetti C, Lucchini F, et al (1975). Cancer mortality in Europe, 2000-2004, and an overview of trends since 1975. Ann Oncol, 21, 1323-60.

Lippka Y, Patschan O, Todenhöfer T, et al (2013). Bladder cancer discussed on the internet: a systematic analysis of gender differences of initial posters on an online discussion board. Springerplus, 2, 445.

Lopez-Beltran A, Sauter G , Gasser T, et al (2004). Tumours of the Urinary System. In: Eble JN, Sauter G, Epstein JI, Sesterhenn IA, editors: World health organization classification of tumours pathology and genetics: tumours of the urinary system and male genital organs, IARC Press, Lyon, pp88-157.

Maruniak NA, Takezawa K, Murphy WM (2002). Accurate pathological staging of urothelial neoplasms requires better cystoscopic sampling. J Urol, 167, 2404-7.

Millán-Rodríguez F, Chéchile-Toniolo G, Salvador-Bayarri J, et al (2000). Primary superficial bladder cancer risk groups according to progression, mortality and recurrence. J Urol, 164, 680-4.

Miyamoto H, Brimo F, Schultz L, et al (2010). Low-grade papillary urothelial carcinoma of the urinary bladder: a clinicopathologic analysis of a post-World Health Organization/International Society of Urological Pathology classification cohort from a single academic center. Arch Pathol Lab Med, 134, 1160-3.

Mostofi FK, Sobin LH and Torloni H (1973). Histological typing of urinary bladder tumours. International Histological Classification of Tumors. No:10. WHO Geneva, Switzerlend, pp9-33.

Negri E and Vecchia CL (2007). Epidemiology and prevention of bladder cancer. In: Bassi PF, Pagano F, editors. Invasive Bladder Cancer. Springer, London, pp1-14.

Nielsen ME, Shariat SF, Karakiewicz PI, et al (2007). Bladder Cancer Research Consortium (BCRC). Advanced age is associated with poorer bladder cancer-specific survival in patients treated with radical cystectomy. Eur Urol, 51, 699-706.

Nomikos M, Pappas A, Kopaka ME, et al (2011). Urothelial carcinoma of the urinary bladder in young adults. Presentation, clinical behavior and outcome. Adv Urol, 480738.

Oosterhuis JW, Schapers RF, Janssen-Heijnen ML, et al (2002). Histological grading of papillary urothelial carcinoma of the bladder: prognostic value of the 1998 WHO/ISUP classification system and comparison with conventional grading systems. J Clin Pathol, 55, 900-5.

Pan CC, Chang YH, Chen KK, et al (2010). Prognostic significance of the $2004 \mathrm{WHO} / \mathrm{ISUP}$ classification for prediction of recurrence, progression, and cancer-specific mortality of non-muscle-invasive urothelial tumors of the urinary bladder: a clinicopathologic study of 1,515 cases. Am J Clin Pathol, 133, 788-95.

Parkin DM, Pisani P, Ferlay J (1999): Estimates of the worldwide incidence of 25 major cancers in 1990. Int J Cancer, 80 , $827-41$.

Quirk JT, Li Q, Natarajan N, Mettlin CJ and Cummings KM (2004). Cigarette smoking and the risk of bladder cancer in men and women. Tob Induc Dis, 2, 141-4.

Rosai J (2011) Bladder. In Rosai and Ackerman's Surgical Pathology. Rosai J editor, tenth ed. Mosby Elsevier, New York, pp1247-71.

Schapers RF, Pauwels RP, Wijnen JT, et al (1994). A simplified grading method of transitional cell carcinoma of the urinary bladder: reproducibility, clinical significance and comparison with other prognostic parameters. Br J Urol, 73, 625-31.

Schned AR, Andrew AS, Marsit CJ, et al (2008). Histological classification and stage of newly diagnosed bladder cancer in a population-based study from the Northeastern United States. Scand J Urol Nephrol, 42, 237-42.

Shephard EA, Stapley S, Neal RD, et al (2012). Clinical features of bladder cancer in primary care. Br J Gen Pract, 62, 598-604.

Siegel R, Ma J, Zou Z, Jemal A (2014). Cancer Statistics, 2014. CA Cancer J Clin, 64, 9-29.

Silverman DT, Hartge P, Morrison AS and Devesa SS (1992). Epidemiology of bladder cancer. Hematol Oncol Clin North Am, 6, 1-30.

Skinner DG (1977). Current state of classification and staging of bladder cancer. Cancer Res, 37, 2838-42.

Sobin LH, Gospodarowicz MK and Wittekind C (2009). TNM Classification of Malignant Tumours, 7Th Ed. International Union against Cancer (UICC). John Wiley \& Sons, Ltd., Atrium, Southern Gate, Chichester, West Sussex UK, pp262-265.

Stanton ML, Xiao L, Czerniak BA and Guo CC (2013) Urothelial tumors of the urinary bladder in young patients: a clinicopathologic study of 59 cases. Arch Pathol Lab Med, 137, 1337-41.

Taylor JA 3rd and Kuchel GA (2009). Bladder cancer in the elderly: clinical outcomes, basic mechanisms, and future research direction. Nat Clin Pract Urol, 6, 135-44.

Van der Heijden AG and Witjes JA (2009). Recurrence, Progression, and Follow-Up in Non-Muscle-Invasive Bladder Cancer. European Urology Supplements, 8556-562.

Vedder MM, Márquez M, de Bekker-Grob EW, et al (2014). Risk prediction scores for recurrence and progression of nonmuscle invasive bladder cancer: an international validation in primary tumours. PLoS One, 9, 96849.

Wang ZH, Li YY, Hu ZQ, et al (2012). Does urothelial cancer of bladder behave differently in young patients? Chin Med $J$ (Engl), 125, 2643-8. 